\title{
Interaction between the microbiome and TP53 in human lung cancer
}

\author{
K. Leigh Greathouse ${ }^{1,14}$, James R. White ${ }^{2}$, Ashely J. Vargas ${ }^{1}$, Valery V. Bliskovsky ${ }^{3}$, Jessica A. Beck1, \\ Natalia von Muhlinen ${ }^{1}$, Eric C. Polley ${ }^{4}$, Elise D. Bowman ${ }^{1}$, Mohammed A. Khan ${ }^{1}$, Ana I. Robles ${ }^{1}$, Tomer Cooks ${ }^{1}$, \\ Bríd M. Ryan ${ }^{1}$, Noah Padgett ${ }^{5}$, Amiran H. Dzutsev ${ }^{6}$, Giorgio Trinchieri ${ }^{6}$, Marbin A. Pineda', Sven Bilke', \\ Paul S. Meltzer ${ }^{7}$, Alexis N. Hokenstad ${ }^{8}$, Tricia M. Stickrod ${ }^{9}$, Marina R. Walther-Antonio ${ }^{8,10}$, Joshua P. Earl ${ }^{11}$, \\ Joshua C. Mell ${ }^{11}$, Jaroslaw E. Krol ${ }^{11}$, Sergey V. Balashov ${ }^{11}$, Archana S. Bhat ${ }^{11}$, Garth D. Ehrlich ${ }^{11}$, Alex Valm ${ }^{12}$, \\ Clayton Deming ${ }^{12}$, Sean Conlan ${ }^{12}$, Julia Oh ${ }^{13}$, Julie A. Segre ${ }^{12}$ and Curtis C. Harris ${ }^{1 *}$
}

\begin{abstract}
Background: Lung cancer is the leading cancer diagnosis worldwide and the number one cause of cancer deaths. Exposure to cigarette smoke, the primary risk factor in lung cancer, reduces epithelial barrier integrity and increases susceptibility to infections. Herein, we hypothesize that somatic mutations together with cigarette smoke generate a dysbiotic microbiota that is associated with lung carcinogenesis. Using lung tissue from 33 controls and 143 cancer cases, we conduct 165 ribosomal RNA (rRNA) bacterial gene sequencing, with RNA-sequencing data from lung cancer cases in The Cancer Genome Atlas serving as the validation cohort.

Results: Overall, we demonstrate a lower alpha diversity in normal lung as compared to non-tumor adjacent or tumor tissue. In squamous cell carcinoma specifically, a separate group of taxa are identified, in which Acidovorax is enriched in smokers. Acidovorax temporans is identified within tumor sections by fluorescent in situ hybridization and confirmed by two separate $16 \mathrm{~S}$ rRNA strategies. Further, these taxa, including Acidovorax, exhibit higher abundance among the subset of squamous cell carcinoma cases with TP53 mutations, an association not seen in adenocarcinomas.

Conclusions: The results of this comprehensive study show both microbiome-gene and microbiome-exposure interactions in squamous cell carcinoma lung cancer tissue. Specifically, tumors harboring TP53 mutations, which can impair epithelial function, have a unique bacterial consortium that is higher in relative abundance in smoking-associated tumors of this type. Given the significant need for clinical diagnostic tools in lung cancer, this study may provide novel biomarkers for early detection.
\end{abstract}

Keywords: Lung cancer, Microbiome, TP53, Squamous cell carcinoma, Mutation

\section{Background}

Lung cancer is the leading cancer diagnosis worldwide (1.8 million/year) and has a higher mortality than that of the next top three cancers combined (158,080 vs 115,760 deaths) [1]. Unfortunately, lung cancer survival remains poor and has shown minimal improvement over the past five decades, owing to diagnosis at advanced stage and resistance to standard chemotherapy [2]. While we have

\footnotetext{
* Correspondence: curtis_harris@nih.gov

${ }^{1}$ Laboratory of Human Carcinogenesis, Center for Cancer, Research, National Cancer Institute, National Institutes of Health, 37 Convent Dr., Rm 3068A, MSC 4258, Bethesda, MD 20892-4258, USA

Full list of author information is available at the end of the article
}

made significant strides with targeted receptor therapy and immunotherapy, biomarkers with higher specificity would improve diagnosis and treatment for these individuals.

Epidemiological evidence indicates an association between repeated antibiotic exposure and increased lung cancer risk; however, the contribution of the lung microbiome to lung cancer is unknown [3]. The first line of defense against inhaled environmental insults, including tobacco smoke and infection, is the respiratory epithelium. Until recently, healthy lungs were regarded as essentially sterile; however, studies now illustrate the presence of a lung microbiota [4], the community of microscopic

(c) The Author(s). 2018 Open Access This article is distributed under the terms of the Creative Commons Attribution 4.0 International License (http://creativecommons.org/licenses/by/4.0/), which permits unrestricted use, distribution, and 
organisms living within the host lung, which is altered in respiratory diseases including asthma, chronic obstructive pulmonary disease (COPD), and cystic fibrosis [5]. Disruption of the epithelium by tobacco smoke can be a primary cause of inflammatory pathology, which is seen in both COPD and lung cancer. Dysbiosis has been observed in both humans and model systems of COPD and cystic fibrosis $[6,7]$. In COPD patients and in vitro, cigarette smoke has been shown to reduce epithelial integrity and cell-cell contact, which can increase susceptibility to respiratory pathogens or other environmental pollutants [8]. Disturbances in the microbiome, from cigarette smoke, epithelial damage, or gene mutations, can allow pathogenic species to dominate the community or increase virulence of other normally commensal microbes. Evidence of this has been demonstrated in patients with cystic fibrosis who have more virulent forms of $P$. aeruginosa [9]. These inflammatory associated events have been proposed to lead to an increased risk or progression of diseases, including lung cancer.

Several bacteria are associated with chronic inflammation and subsequent increased risk of lung and colon cancer, including Mycobacterium tuberculosis (lung cancer) [10], Bacteroides fragilis, and Fusobacterium nucleatum (colon cancer) [11]. Recent microbiome studies in colon cancer have demonstrated a contribution of bacteria to carcinogenesis. Specifically, F. nucleatum, a bacterium commonly isolated from patients with inflammatory bowel disease, may be a risk factor for colon cancer $[11,12]$. The more virulent strains of $F$. nucleatum affect colon cancer progression in animal models and increase tumor multiplicity [13] by various mechanisms including favoring the infiltration of tumor-promoting myeloid cells to create a pro-inflammatory environment [14]. Colorectal carcinomas associated with high abundance of fecal $F$. nucleatum were found to have the highest number of somatic mutations, suggesting that these mutations create a pathogen-friendly environment [15]. Similarly, B. fragilis can secrete endotoxins that cause DNA damage leading to mutations and colon cancer initiation [16]. Furthermore, the loss of the oncogenic protein p53 in enterocytes impairs the epithelial barrier and allows infiltration of bacteria resulting in inflammatory signaling (NF-kB), which is required for tumor progression [17]. The tumor suppressor gene TP53 is the most commonly mutated gene in lung cancer [18], with certain missense mutations showing gain of oncogenic function [19]; however, the relationship between TP53 and microbiota in lung cancer remains unknown. Herein, we hypothesize that somatic mutations together with environmental exposures are correlated with tissue-associated alterations in the microbial community of the lung, which may participate in lung carcinogenesis.

\section{Results}

To investigate the lung mucosal-associated microbial alterations in the etiology of lung cancer, we analyzed samples from the NCI-MD case-control study $(n=143$ tumor and $n=144$ non-tumor adjacent tissues) and lung cancer samples from The Cancer Genome Atlas (TCGA; $n=1112$ tumor and non-tumor adjacent RNA-sequencing [RNA-seq] data from tissues) for validation. In addition, we used the clinical information from these two sample populations to control for confounders in lung cancer risk and progression (age, gender, smoking, race, family and medical history, and co-morbidities), as well as factors that are known to alter the human microbiome (antibiotics and neoadjuvant therapy). Given the paucity of healthy lung tissue available for study, we utilized two separate tissue biorepositories. Non-cancerous lung tissue was obtained by lung biopsy from individuals with benign lung nodules without cancer or non-cancer lung from immediate autopsy [20], which was used as a referent control (Table 1).

Given the high potential for contamination in low-biomass samples, such as the lung, we took several measures to address this issue controlling for contamination points in the collection process. To assess possible confounding with sequence quality, we conducted sequencing quality control analysis by Phred score and by sequencing run (Additional file 1: Figure S1). In order to remove possible contaminants from our analysis, we first performed a threshold analysis similar to a previous study [21], wherein we plotted the mean percent abundance across experimental samples versus negative control samples and removed those that were $\geq 5 \%$ in both experimental and negative control samples (Additional file 1: Figure S2). We next applied a statistical analysis wherein we used a systematic removal process of putative contaminants including Herbaspirillum, Halomonas, and Shewanella (Additional file 1: Table S1). At each stage of removal, we report the number of Mann-Whitney $p$ values $<0.05$ comparing paired tumor normal samples showing the greatest rise the number of significant $\mathrm{p}$-values with the removal top five contaminants (Additional file 1: Table S1). At each stage of removal, we report the number of Mann-Whitney $p$ values $<0.05$ comparing paired tumor normal samples showing the greatest rise the number of significant $p$ values with the removal top five contaminants (Additional file 1: Table S1). Additionally, we conducted hierarchal clustering of negative controls, non-tumor samples, and tumor samples independently in order to visualize and identify the strongest sources of contamination (Additional file 1: Figures S2 and S3). The combination of these analyses resulted in initial removal of the genera Halomonas, Herbaspririllium, Shewanella, Propionibacterium, and Variovorax. 
Table 1 Descriptive summary of population samples

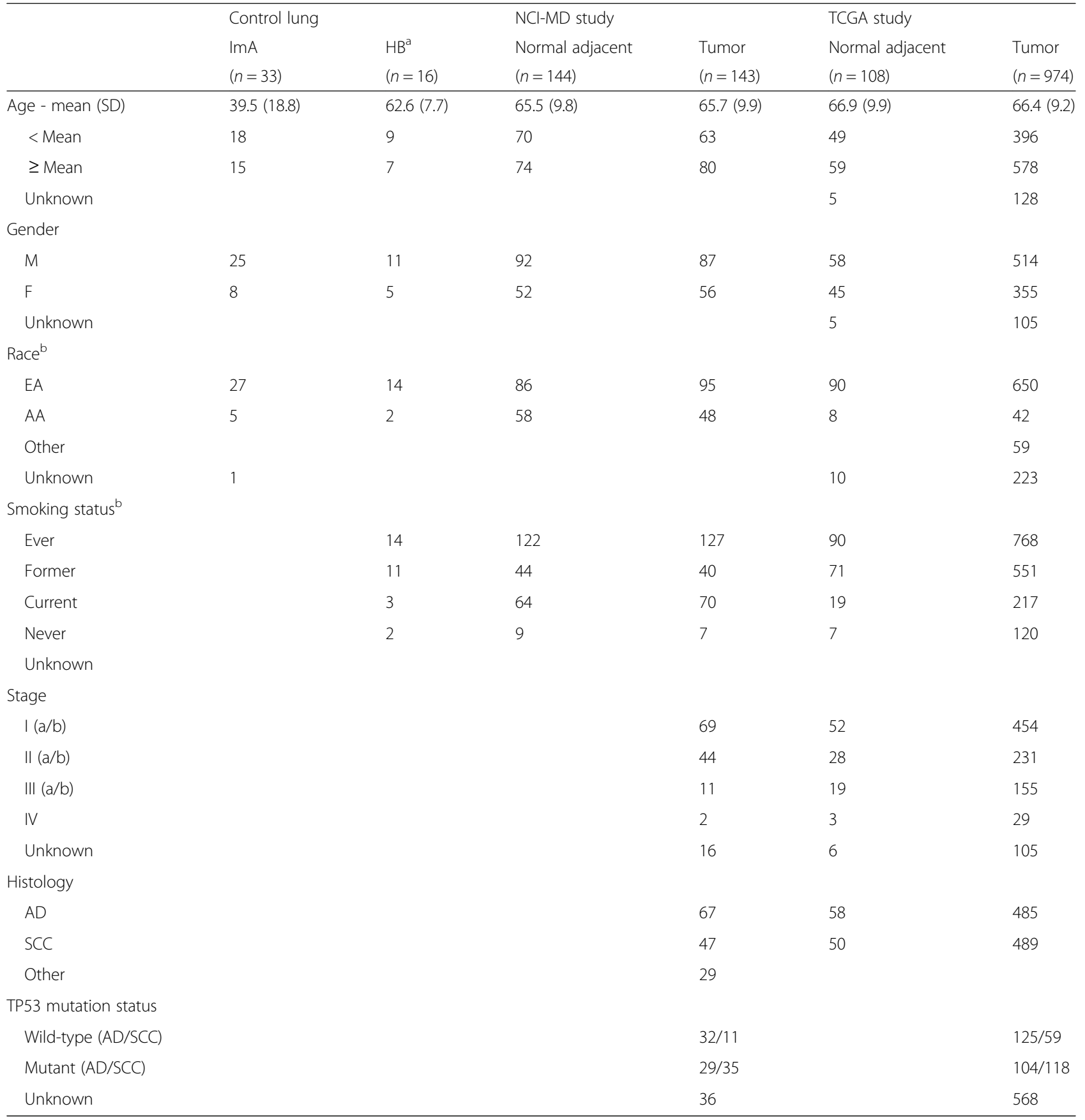

${ }^{\text {aTwo }}$ cases removed due to emphysema

${ }^{\mathrm{b}}$ Smoking status and race self-reported

ImA immediate autopsy, $H B$ hospital biopsy

To identify the microbial communities present in each tissue type, we sequenced the V3-V5 $16 \mathrm{~S}$ ribosomal RNA (rRNA) bacterial gene using the Illumina MiSeq platform. After quality filtering and contaminant removal, 34 million quality sequences were retained for operational taxonomic unit (OTU) clustering and downstream analysis (Additional file 1: Table S2).
To enable us to validate findings from our NCI-MD $16 \mathrm{~S}$ rRNA gene sequencing analysis, we took advantage of the TCGA lung cancer database. Using the unmapped RNA-seq reads from these samples $(N=1112$ and $n=$ 106 paired tumor/non-tumor), we analyzed with our metagenomics analysis pipeline. After removal of all human reads, we took the remaining non-human reads 
and used three separate tools, MetaPhlAn, Kraken, and PathoScope, to assign reads to taxonomy, including bacteria, virus, and fungi (Additional file 1: Table S2). Due to the highly curated database of PathoScope, we were able obtain to species and in some cases strain-level putative identification of RNA-seq reads. For this reason, and due to its rigorous validation in other studies [22], we used these data as our validation dataset. Unfortunately, given that all patients in this database had lung cancer, we could not validate our microbial findings in non-diseased lung tissue in the TCGA dataset. Given that this was one of the first times TCGA was used to completely profile the microbiota of lung cancer, we asked how similar the 16S rRNA gene sequencing and RNA-seq microbial communities were at the phylum and genus levels. Using an overall threshold of $0.01 \%$ of genus level abundance, we identified 236 overlapping genera out of 520 total genera in the $16 \mathrm{~S}$ rRNA gene sequencing data and 609 total genera in the RNA-seq data (Additional file 1: Figure S4).

\section{Bacterial profile of the lung cancer microbiome is dominated by Proteobacteria and validated in a separate lung cancer data set}

We know from previous microbial studies of lung disease that bacterial composition shifts occur compared to normal non-diseased lungs [23] and associated with disease severity [24]; however, these compositional changes have not been examined in lung cancer. In order to identify the microbial changes associated with lung cancer, we first examined the ecological diversity within samples (alpha diversity) and between samples (beta diversity) of non-cancerous (immediate autopsy and hospital biopsy) tissues, non-tumor adjacent (NT) and tumor (T) tissues from 16S rRNA gene sequencing. At the phylum level, we observed increases in Proteobacteria (Kruskal-Wallis $p=0.0002$ ) and decreases in Firmicutes (Kruskal-Wallis $p=0.04$ ) in lung tissue hospital biopsies, as well as in tumor and associated non-tumor tissues from the NCI-MD study compared with non-cancer population control lung tissues, as has been seen in COPD [25] (Fig. 1a). Further, we note higher Fusobacterium in ImA and $\mathrm{HB}$ controls as compared to cancer cases, though it is unclear what this finding indicates at the phylogenic level. We also observed a similar increase in Proteobacteria (Mann-Whitney $p=0.02$ ) between non-tumor lung tissue and lung cancer in the TCGA study, indicating that this is recurrent phenomenon in lung cancer (Fig. 1a). However, the lack of similarity between the NCI-MD and TCGA non-tumor samples may be attributed to the TCGA data being derived from multiple sample populations in the United States, differences in sample prep and in sequencing platforms, as illustrated by Meisel et al. [26].
To identify ecological diversity changes associated with lung cancer, we next examined the richness (Chaol) and diversity (Inverse Simpson) of the microbiome within samples (alpha diversity) of non-disease (immediate autopsy and hospital biopsy) lung tissues, non-tumor adjacent tissues, and tumor tissues from 16S rRNA gene sequencing (NCI-MD study). Specifically, Chaol measurement demonstrated a significant increase in both tumor and non-tumor tissue richness as compared to immediate autopsy control tissue samples (Fig. 1b). Similarly, using the Inverse Simpson index, which measures number (richness) and abundance (evenness) of species, we observed a significant increase in alpha diversity in both tumor and non-tumors as compared to hospital biopsy control tissues (Fig. 1b), similar to studies of severe COPD [27], indicating that microbial diversity of lung cancer tissues is altered from its non-diseased state. When we examined tissue from cancer cases, alpha diversity was significantly different between tumor and non-tumors in the NCI-MD study and TCGA study, but results were not consistent between studies or diversity metrics (Additional file 1: Figure S5). However, we did not see any significant changes in alpha diversity by smoking status (never, former, or current) nor correlation with time since quitting smoking (Additional file 1: Figure S4), in cancer-free or lung cancer tissues as has been demonstrated in other lung microbiome studies [28, 29].

We also asked whether there were differences between microbial communities using beta diversity (Bray Curtis). Since we were comparing between studies and between types of sequencing (16S rRNA and RNA-seq), we used a method that could be commonly applied between studies, which excludes phylogeny (e.g. Bray Curtis). Within the NCI-MD study, we observed significant differences in beta diversity between all tissue types (PERMANOVA $F=2.90$, $p=0.001$ ), tumor and non-tumor (PERMANOVA $F=$ 2.94, $p=0.001$ ), and adenocarcinoma (AD) versus squamous cell carcinoma (SCC) (PERMANOVA $F=2.27$, $p=0.005$ ), with tumor vs. non-tumor having the largest among-group distance denoted by the higher $F$ value (Fig. 1c-e). Similarly, we observed significant difference in beta diversity between tumor and non-tumor (PERMANOVA $F=3.63, p=0.001$ ) and AD v SCC (PERMANOVA $F=27.19, p=0.001$ ) (Fig. 1f, g). Together, these data illustrate a trend of increasing diversity and richness associated with lung cancer.

\section{A distinct group of taxa are enriched in squamous cell carcinoma with Acidovorax more abundant in smokers} The two most common types of non-small cell lung cancer are $\mathrm{SCC}$ and $\mathrm{AD}$, arising centrally from the cells lining the bronchi and from peripheral airways, respectively. Previous studies report that the microbial community differs between the bronchi and lower lungs in COPD [6]. This 


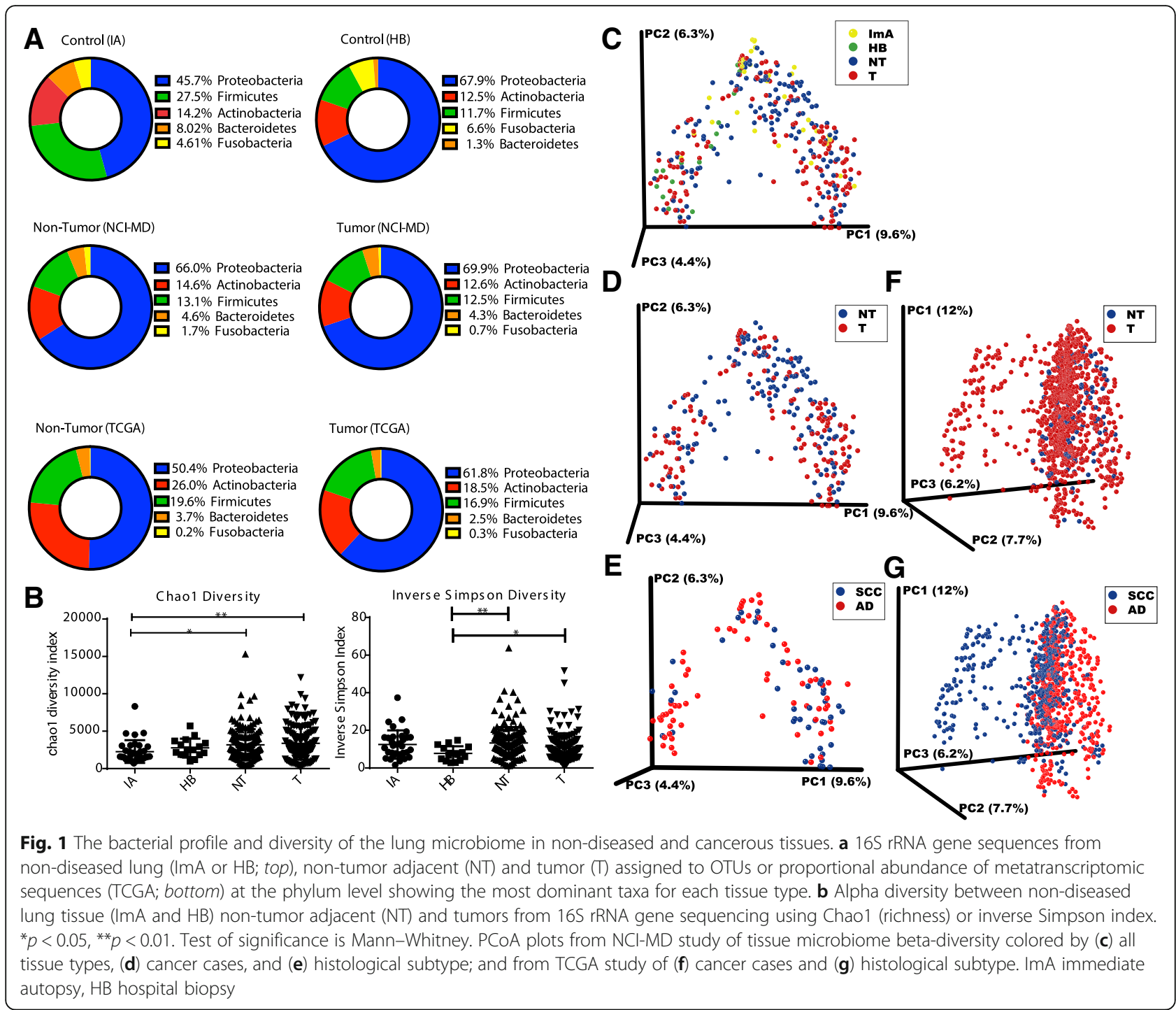

phenomenon of anatomic-specific microbial variation was also apparent in the abundance of genera between bronchial and SCC tumors from the upper lungs with higher abundance of Acidovorax in comparison to AD tumors (Additional file 1: Figure S6). Further, the taxonomic distribution in $\mathrm{AD}$ tumors appears more similar to the taxonomic abundance in COPD, which is generally dominated by Pseudomonas [6]. Given this distinction, we controlled for this potential confounder of lung location in subsequent analyses. This led us to investigate the specific taxonomic pattern further and ask if there was a specific microbial consortia that is enriched in SCC or AD tumor tissue. In the NCI-MD study, we identified 32 genera that were differentially abundant in SCC $(n=47)$ versus $\mathrm{AD}(n=$ 67) tumors (Student's t-test; MW $P<0.05$ ), nine of which were significant after multiple testing correction (FDR) (Acidovorax, Brevundimonas, Comamonas, Tepidimonas, Rhodoferax, Klebsiella, Leptothrix, Polaromonas,
Anaerococcus) (Fig. 2a). We also validated these same observations in the TCGA dataset $(\mathrm{AD}=485, \mathrm{SCC}=489)$ (Mann-Whitney FDR corrected $p$ value< 0.05 ) (Fig. 2b). To control for potential confounders of this association, including age, gender, race, smoking, anatomical location, and stage, we conducted adjusted logistic regression analysis in the NCI-MD study for each taxa separately and confirmed 6/9 of these genera were significantly associated with increased odds of being SCC as compared to AD lung cancer (Fig. 2c, Additional file 1: Tables S5 and S7). Though we had reduced power, we asked whether the time since quitting smoking would change this association, and found that Acidovorax, Klebsiella, Tepidimonas, Rhodoferax, and Anaerococcus remained significant. When we examined the larger TCGA dataset, we also found significantly increased odds of being SCC as compared with AD among 4/9 (Acidovorax, Klebsiella, Rhodoferax, Anaerococcus) of the same genera in adjusted models 


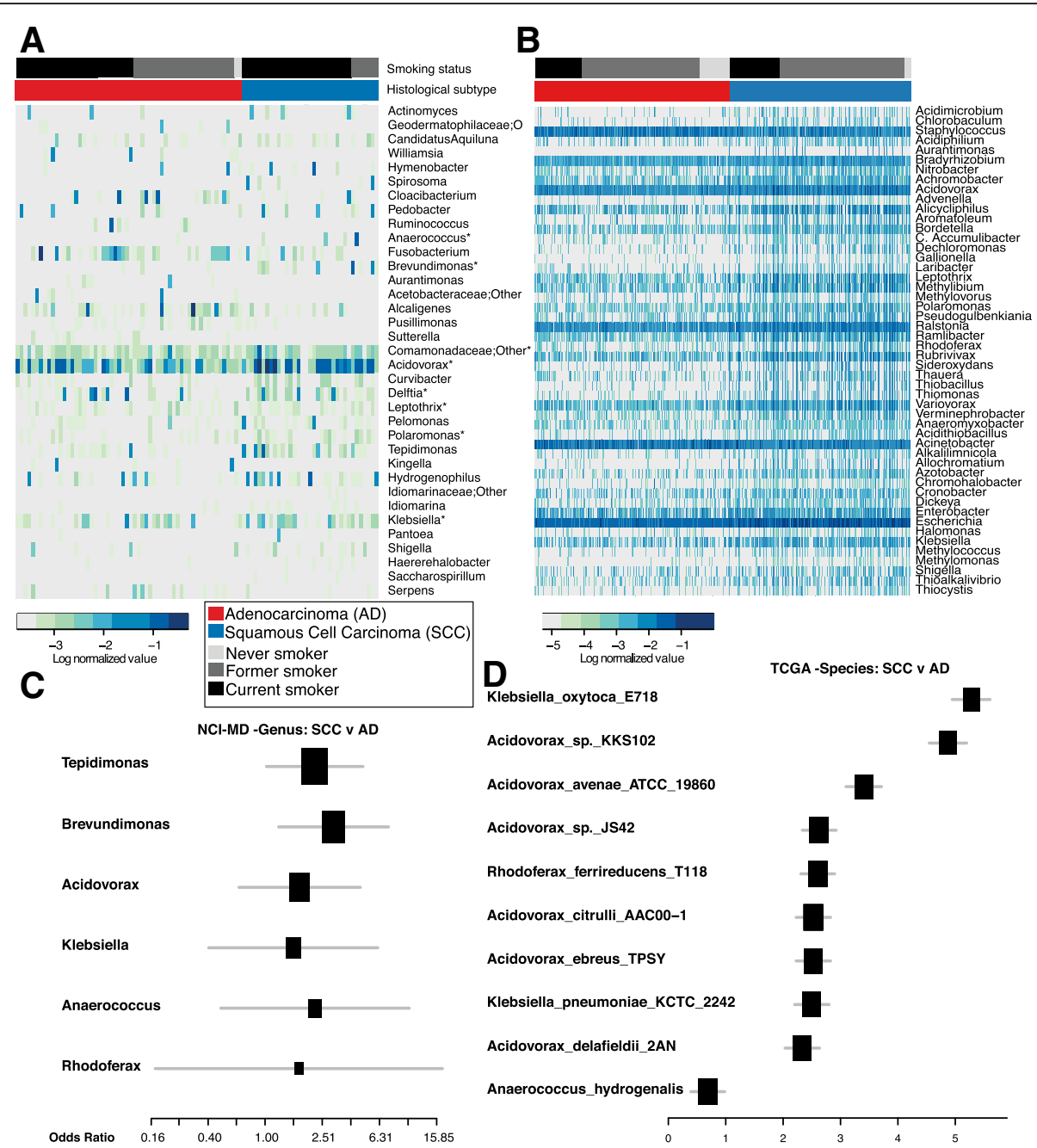

Fig. 2 Taxonomic consortia differentiating smoking status and histological subtype of lung cancer. a Heat maps showing top differentially abundant genera (NCI-MD) (Mann-Whitney $p$ value < 0.05 ; * overlapping between NCI-MD and TCGA) between AD and SCC lung cancer tissue sorted by histological subtype and smoking status. b Heat map showing genera (TCGA) that that are differentially abundant between AD and SCC (Mann-Whitney FDR corrected $p<0.05$ ), sorted by histological subtype and smoking. c Forest plot of odds ratios for genera in NCl-MD dataset that are significantly associated with SCC compared to AD in tumors (adjusted odds ratio $p<0.05$ ). $\mathbf{d}$ Forest plot of odds ratios for species in the TCGA dataset that are significantly associated with SCC vs AD in tumors (adjusted odds ratio FDR corrected $p<0.05$ )

(FDR corrected $P<0.05$ ) (Fig. 2d, Additional file 1: Tables S6 and S8). This association also remained significant after adjusting for pack years and time since quitting smoking. Together these data, validated in two separate cohorts, demonstrate that a specific community of taxa is more abundant in SCC as compared with $\mathrm{AD}$ lung cancer tissue, and are capable of distinguishing between $\mathrm{AD}$ and SCC tumors from individuals with similar exposure to cigarette smoke. However, whether this is a cause or consequence of the development of SCC cancer remains unknown.

Both SCC and AD lung cancers are associated with smoking; however, the association between smoking and SCC is stronger [30], which leads us to ask whether any of the SCC-enriched taxa were also associated with smoking. We stratified the tumor samples into never smokers $(n=7)$ or ever-smokers (current $[n=70]$ and former smokers [ $n=$ 40]) using linear discriminant analysis (LEfSe) to identify smoking-associated microbial biomarkers in SCC tumors. We identified six genera that were able to distinguish ever (former and current) versus non-smokers in our NCI-MD study (Acidovorax, Ruminococcus, Oscillospira, Duganella, Ensifer, Rhizobium) (Additional file 1: Figure S6C). Specifically, Acidovorax was more abundant in former and current smokers as compared with never smokers (Kruskal-Wallis $p$ value $<0.05$ ) (Fig. 3a), with a similar trend observed in the TCGA dataset $\left(\mathrm{n}_{\text {never }}=120, \mathrm{n}_{\text {former }}=551, \mathrm{n}_{\text {current }}=217\right)$ (Kruskal-Wallis $p=0.27$; ANOVA $p=0.02$ ). We did not, however, observe any correlation between Acidovorax abundance and smoking time cessation. Interestingly, the relative abundance of Acidovorax and Klebsiella were 

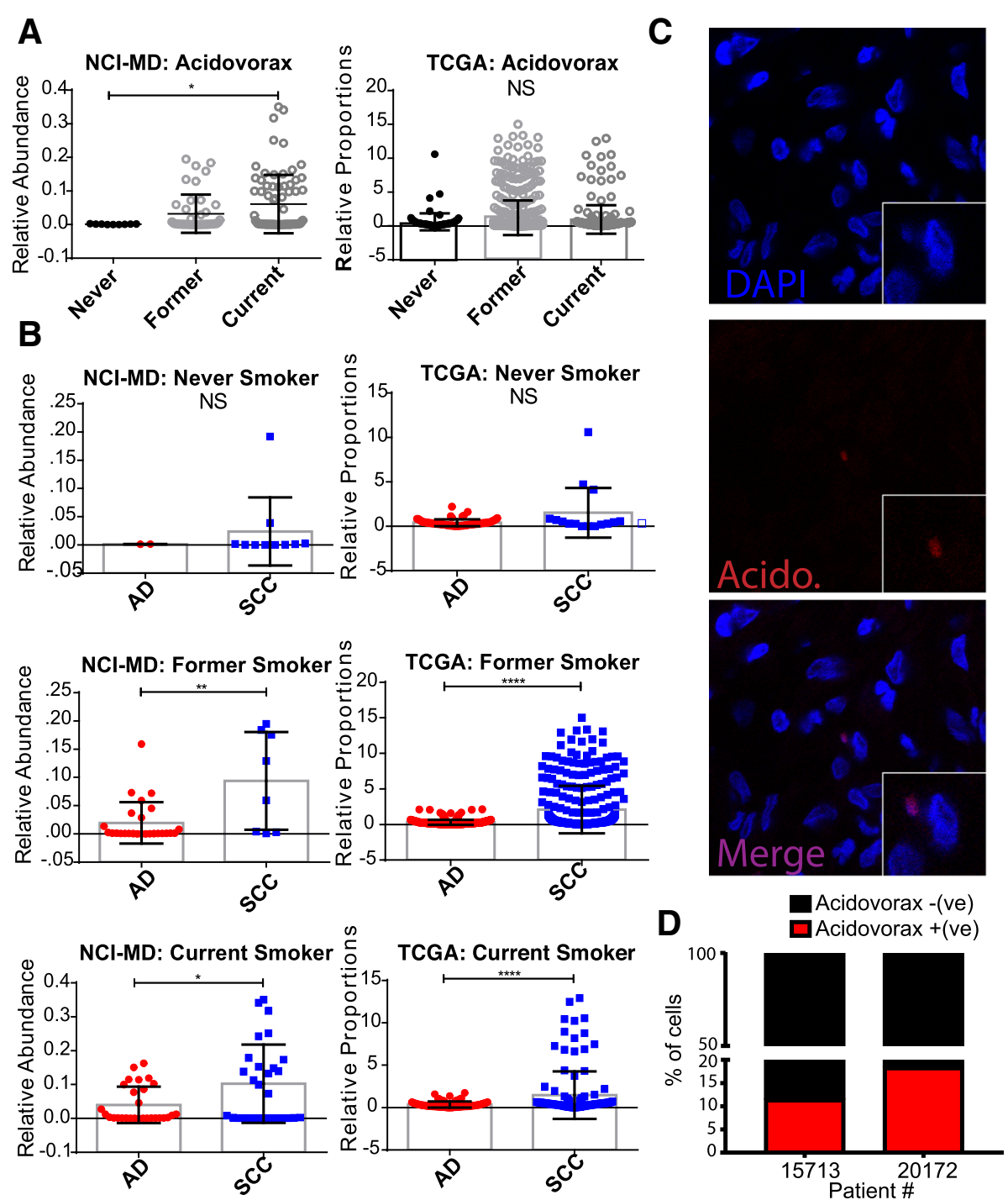

Fig. 3 Relative abundance of Acidovorax stratified by smoking status and histological subtype. a Relative abundance of Acidovorax stratified by smoking status in the NCI-MD (left) and TCGA (right) datasets. b Relative abundance of Acidovorax in never, former, and current smokers stratified by histological subtype in the NCI-MD (left) and TCGA (right) datasets. c Representative FISH images of tumor tissue sections using fluorescent probe specific to Acidovorax. d Quantification of Acidovorax probe reactivity (10 fields; at least 300 cells counted) showing percentage (\%) of cells with perinuclear probe reactivity from two lung cancer cases (15,713 - SCC/current smoker; 20,172 - SCC/former smoker). ${ }^{*} p<0.05,{ }^{* *} p<0.01$, ${ }^{* * * *} p<0.0001$. Tests of significance are Mann-Whitney or Kruskal-Wallis and Dunn's multiple comparisons test. NS non-significant

higher in former and current smokers when we stratified by histological subtype in both the NCI-MD and TCGA datasets (Fig. 3b, Additional file 1: Figure S7), indicating not only are there bacteria which have a higher relative abundance in tumors from individuals who smoke, but SCC tumors from smokers have even greater relative abundance of these bacteria. We also demonstrated the presence of this bacterium in lung tumors using FISH (Fig. 3c, d, Additional file 1: Figure S8, Additional file 2), and using PacBio sequencing, which identified the species as $A$. temperans (Additional file 1: Table S4). We did not find any significant associations between pack years or time since quitting smoking and the abundance of these taxa in either study among SCC tumors in either study.

TP53 mutations are associated with enrichment of SSCenriched taxa

The most prevalent somatic mutation in SCC lung tumors is in the gene TP53 [31]. Previous studies demonstrate that mutations in TP53, specifically in colon cancer, lead to disruption of the epithelial barrier allowing the infiltration of tumor-foraging bacteria and resulting in disease progression [17]. Given that TP53 mutations are found in $75-80 \%$ of SCC tumors, we hypothesized that these 
SCC-associated taxa may be more abundant in tumors with TP53 mutations, owing to the loss of the epithelial barrier function in these tumors. To address this question, we investigated the association between TP53 mutations in both the NCI-MD $(n=107)$ and TCGA $(n=409)$ datasets using either TP53 specific sequencing (MiSeq) or the published TP53 mutation analysis data from TCGA [31]. We first analyzed all tumors in the NCI-MD study regardless of histology and identified a group of taxa that were more abundant in tumors with TP53 mutations (Fig. 4a). To have greater power, we performed the same analysis in the TCGA dataset and observed a significant increase in these same taxa (MW FDR corrected $P<0.05$ ) (Fig. 4b). When analyzing only SCC tumors $(n=46)$, this signature became stronger in tumors with TP53 mutations in both datasets, specifically among the SCC-associated taxa previously identified (Fig. 4c, d). In the NCI-MD study, we found that $5 / 9$ of the genera (Acidovorax, Klebsiella, Rhodoferax, Comamonas, and Polarmonas) that differentiated SCC from AD were also more abundant in the tumors harboring TP53 mutations, though not statistically significant (Fig. 4c). In the TCGA dataset, the fold change in all five SCC-associated genera were significantly higher in SCC tumors $(n=177)$ with TP53 mutations (MW corrected FDR < 0.01; Fig. 4d). Furthermore, using these same SCC-associated taxa we observed no pattern of association in AD tumors with TP53 mutations indicating this signature was specific to SCC with TP53 mutations (Additional file 1: Figures S9A and S9B). Overall, these data are consistent with the hypothesis that mutations in TP53 are associated with the enrichment of a microbial consortia that are highly represented in SCC tumors.

\section{Discussion}

Gene-environment interactions have been identified as contributors to cancer incidence [32]; however, little is known about gene-microbiome interactions in carcinogenesis. We demonstrate a gene-microbiome association in human lung cancer as well as histological evidence of a smoking-associated bacterium, Acidovorax. Herein, we identify a microbial consortia that is associated with a histological subtype of lung cancer, SCC, which is further enriched in tumors with mutations in TP53. Given the strong association between smoking and development of SCC, it follows that a subgroup of this SCC consortium would also be found in smoking-associated SCC. We validate this assumption finding Acidovorax spp. more abundant in SCC tumors harboring TP53 mutations and confirmed the presence of this genus histologically. These results suggest that smoking together with tumorigenesis may provide an environment conducive to the growth of Acidovorax spp. and similar species, which can flourish in nutrient-depleted environments, such as that of the lung. Collectively, these observations indicate that a state of dysbiosis exists in lung cancer. The hypothesis generated is that epithelial cells in the lung exposed to tobacco smoke and/or mutations in TP53 are invaded by species that take advantage of this new microenvironment, suggesting these bacteria could act as promoters in lung tumorigenesis.

Several cancers are caused by bacteria and viruses, including cervical cancer (HPV), liver cancer (HBV), and gastric cancer (H. pylori and potentially B. fragilis); however, very few microbes have been identified as carcinogenic. Beyond acting as initiators, other relationships exist between microbe and host tissue environments, in a similar manner to chemical carcinogens. These relationships include bacteria that act as promoters and those that are just passengers in the tumorigenesis process. While this study is not longitudinal, our data suggest the latter two possibilities, either they are promoters or passengers.

In support of the promoter hypothesis, it is plausible that smoking creates an environment that allows these bacteria to outcompete other species for resources and thus survival, which allows exposure to microbial factors enhancing tumorigenesis. Smoking is most strongly associated with the SCC histological subtype of lung cancer; however, whether smoking alters the lung tissue microbiome is still not well understood, especially in the context of disease. Multiple studies using various samples tissue and non-tissue types (e.g. oral and/or nasal swabs, bronchial lavage fluid, or lung tissue) have found inconsistent results in alpha diversity by smoking status. From our study, while we did not observe differences in alpha diversity, we observe a significant difference in the taxonomic consortia among smokers as compared to non-smokers, specifically in Acidovorax and Klebsiella spp. Similarly, oral and nasal microbial taxa differences have been observed between smokers and non-smokers [29, 33]. From a large study of the naso- and oropharynx, significant differences in specific microbial taxa were identified between smokers and non-smokers [34]. Additionally, in a study of non-malignant lung tissue $(n=152)$, they observed a significant increase in alpha diversity with higher number of pack years of smoking [35]. While they identified Acidovorax, Anaerococcus, and Comamonas in smokers, these taxa did not differentiate smokers and non-smokers in a healthy population. However, in a recent study of non-malignant lung tissue, which compared tissue to isolated extracellular vesicles (EVs) from tissues, the greater diversity was identified specifically in EVs, with a greater abundance of Acidovorax specifically found in the EVs of smokers, indicating a possible factor in differential findings observed among previous studies [36].

These data indicate that smoking alone may be insufficient to alter the microbial population in a healthy population. However, smoking has been shown to suppress the immune system and induce epithelial barrier dysfunction 


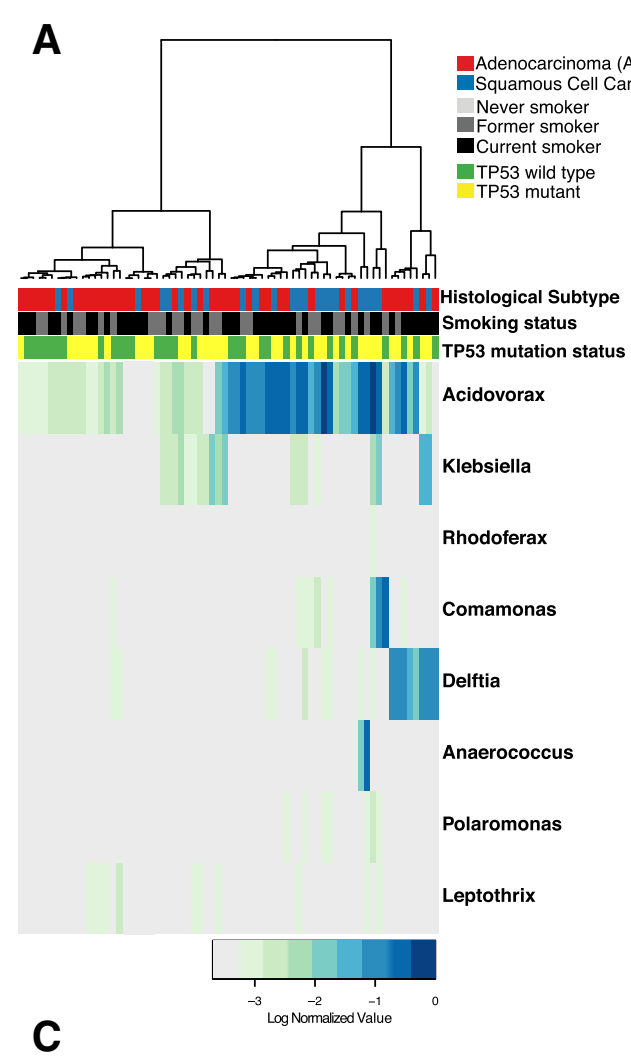

\section{B}

Boma (SCC)

Never smoker

Former smoker

TP53 wild type
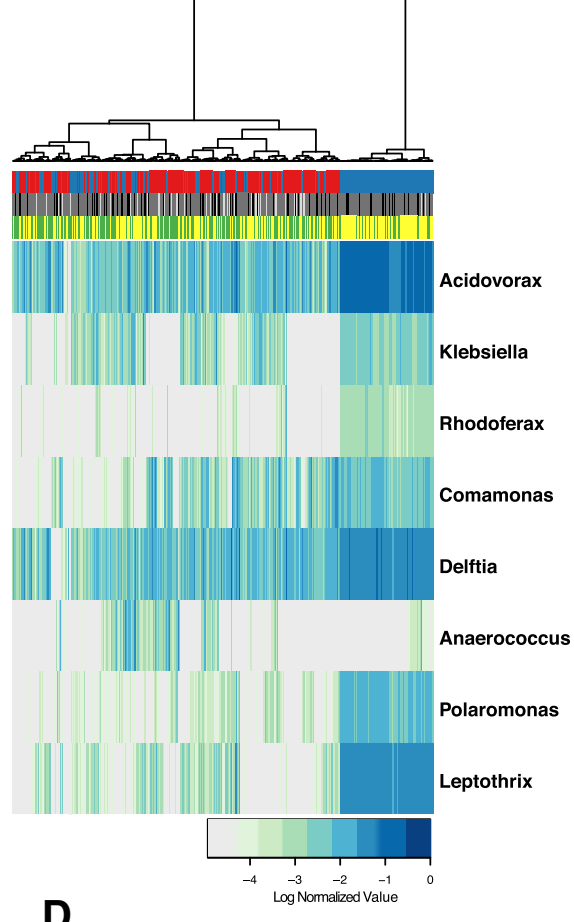

NCI-MD: TP53 Mutation vs Wild Type

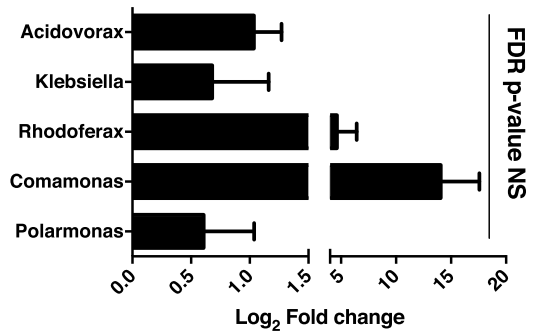

TCGA:TP53 Mutation vs Wild Type

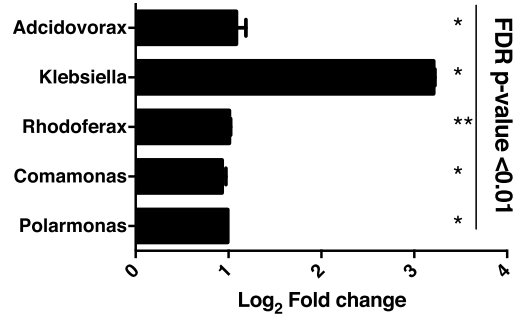

Fig. 4 Mutations in TP53 associated with abundance of taxonomic signature specific to squamous cell lung tumors. a Heat map of genus-level abundance in NCl-MD data colored by mutation status, TP53 wild-type or mutated, smoking, and histological subtype in all lung tumor samples. b Heat map of genus-level abundance from TCGA data in all tumors colored by mutation status, TP53 wild-type or mutated, smoking, and histological subtype. c, $\mathbf{d}$ Fold change in mean abundance of SCC-associated taxa in NCI-MD or TCGA tissues comparing TP53 mutated to wildtype. Test of significance is Mann-Whitney. Fold change among all taxa in (d) are significant after FDR correction $<0.01$. $\left(\mathrm{NCI}-\mathrm{MD} ; \mathrm{SCC}_{\mathrm{wt}}=11\right.$, $\mathrm{SCC}_{\mathrm{mut}}=35$ and TCGA; $\left.\mathrm{SCC}_{\mathrm{wt}}=59, \mathrm{SCC}_{\mathrm{mut}}=118\right)$

[37]. Specifically, Acidovorax spp. have been identified in two common brands of cigarettes [38] and have the capacity to metabolize multiple organic pollutants like those found in cigarette smoke [39]. Therefore, degradation of tobacco smoke compounds, such as polycyclic aromatic hydrocarbons by Acidovorax spp., may promote survival of transformed cells and subsequently tumor promotion. These factors may allow taxa direct access to epithelial cells where microbial toxins or reactive oxygen/nitrogen from the aforementioned species to directly or indirectly encourage malignant transformation of the lung epithelium via
DNA damage and mutations in TP53 [40-42]. Once the epithelial barrier defense is lost as a consequence of mutations in TP53 and malignant transformation, these species then may become tumor-foraging bacteria. In support, several bacterial species have been shown to modulate the tumor-suppressor p53 at both the protein and DNA level [43]. Specifically, the loss of p53 in enterocytes in murine models impairs the epithelial barrier and allows infiltration of bacteria resulting in NF- $\mathrm{kB}$ signaling, which was required for tumor progression [17]. This evidence suggests that SCC tumors with TP53 mutations could have poor 
epithelial barrier function, thus allowing tumor foraging bacteria, such as those identified in our study, to become more abundant in tumors with TP53 mutations. The counterfactual is also possible. Similar to the $B$. fragilis toxin ETBF, which is genotoxic and initiates colon carcinogenesis in animal models [44], one or more of the tumor-associated species may induce TP53 mutations. Notably, individuals harboring mutations in TP53 with stage I SCC also have poorer prognosis [45], thus it will be important to determine if any of the species enriched in SCC are functionally related to reduced survival or simply biomarkers of a diminished mucosal barrier function. Whether any of these bacteria are promoting SCC tumorigenesis or inducing mutations in TP53 is currently under investigation.

In support of the passenger hypothesis, our study indicates that smoking is associated with alterations in relative abundance of species in SCC tumors. The number one risk factor for lung cancer is tobacco exposure and is a known factor in chronic lung inflammation. Tobacco and cigarette smoke contain bacterial products (i.e. LPS) that can cause inflammation, impaired barrier function, and potentially alter the microbiome to influence lung carcinogenesis [8, 46, 47]. Additionally, tobacco leaves harbor both mold and potentially pathogenic bacteria that can be transferred in a viable form into the respiratory tract on tobacco flakes inhaled in mainstream smoke [46, 47]. Further, biologically significant quantities of bacteria are microaspirated daily in healthy individuals [48] and thus is possible for these species to accumulate in a pathogen-friendly environment but may not ultimately contribute to tumorigenesis. Nevertheless, future studies should address this issue mechanistically.

The strength of our findings includes the large number of individuals sampled in this study, use of two separate sample populations, two sets of control populations, two separate sequencing methodologies (MiSeq and PacBio), and microscopic validation (FISH) of the species in lung tumor tissue. We have also been diligent in assessing the possibility of contaminating taxa being an artifact of sample collection or sample processing by extensive quality control analysis of sequencing, sequencing across two different platforms, and microscopy. Given the low biomass of these samples, however, we were not able to completely eliminate all contaminants and acknowledge that this may skew the results. While we were able to control for antibiotic exposure in the NCI-MD study, we acknowledge a limitation of the validation study is the inability to control for antibiotic exposure in the TCGA dataset and ImA controls, as well as, significant differences in clinical features between the cancer cases and controls, which could be confounders. However, in a recent study of the microbiome of endoscopic gastric biopsies, confirmation of multiple shared bacteria in clinical samples, specifically $H$. pylori, was demonstrated using the
TCGA RNA-seq data with methods similar to those presented in our study [49].

\section{Conclusions}

With the majority of lung cancer being diagnosed at a late stage, the recent advancement in the treatment of late stage (III/IV) lung cancer with immune checkpoint inhibitors targeting PD-1, nivolumab, has resulted in a $40 \%$ reduced risk of death as compared to standard chemotherapy [50]. The response rate, however, is still not complete for these patients. Important insights into understanding the differential response rates of this new immunotherapy has suggested the composition of the lung microbiome before therapy as a key player in therapeutic effectiveness [51]. Given our results demonstrating alterations in the microbial composition in lung cancer that are histology and mutation specific, future studies should address whether the lung or nasal microbiome composition improves the stratification of patients who would be most responsive to immunotherapy. This suggestion is supported by recent animal studies demonstrating the contribution of the gut microbiome to the effectiveness of immunotherapy [52]. With these results, we foresee a new avenue for mechanistic studies to address the role of microbe-host relationship in lung cancer inflammation, response to therapy, and microbial engineering for drug delivery.

\section{Methods}

\section{Sample populations and datasets}

Samples used for DNA extraction, polymerase chain reaction (PCR) and sequencing were obtained from the ongoing NCI-MD study (seven hospitals participating in the greater Baltimore, MD area recruited during 19992012), as described previously [53], from which 398 lung cancer cases were obtained, and included both tumor and non-tumor adjacent, with 121 matched pairs. The final sample set used for analysis after sequencing, which contained 106 matched pairs after quality control, is found in Table 1. Lung tumors and paired non-tumor adjacent samples from the NCI-MD study were obtained at the time of surgery, from which a section of tumor and non-involved adjacent lung tissue from the same lung resection were flash frozen and stored at $-80{ }^{\circ} \mathrm{C}$, with an estimated time to cold ischemia of $66 \mathrm{~min}$. At the time of study entry, a detailed patient interview was conducted to obtain basic clinical information in addition to previous cancers, neoadjuvant therapies, current medications, family history of cancer, smoking history, education level, and financial status. Staging was assigned using the Cancer Staging Manual of the American Joint Committee on Cancer (AJCC) 7th edition. Preoperative antibiotics were administered for those cases recruited after 2008 and any antibiotic oral medication use was controlled for as a covariate for all statistical analysis in model testing; 
however, these data were not available for immediate autopsy $(\operatorname{ImA})$ non-cancer samples. Controls representing non-cancerous tissue were obtained from the Lung Cancer Biorepository Research Network ( $n=16$; hospital controls). Theses samples were obtained as frozen lung specimens from individuals who had a previous positive nodule identified by PET scan and subsequently underwent tissue biopsy, which was ruled benign. The average non-operative ischemia time was $34 \mathrm{~min}(16-70 \mathrm{~min})$ for these samples. Clinical information included those listed above as well as smoking history, antibiotic usage $(\mathrm{Y} / \mathrm{N})$, and disease diagnosis. Two cases had emphysema at the time of biopsy and were not used in the analyses. Immediate autopsy $(\operatorname{ImA})$ samples obtained from the University of Maryland (UMD) hospital, which is part of the NCI-MD study population $(n=41$; population controls) (Table 1). Lung tissue from ImA was received frozen from the UMD biorepository and served as the population controls for non-cancer lung tissue. Briefly, samples from ImA were obtained within minutes $(<30 \mathrm{~min})$ after death and put on ice for $<30 \mathrm{~min}$ during dissection before cold ischemia at $-80^{\circ} \mathrm{C}$. All ImA subjects underwent extensive autopsy and were determined to be cancer-free. Demographic information included age, gender, race, and cause of death only. Non-smokers in the NCI-MD study were categorized as having smoked $<100$ cigarettes or $<5$ packs over a lifetime, whereas smokers were categorized as current smokers or formers smokers, who had quit for $>6$ months. Sequences derived from RNA-seq of lung tumor $(n=1006)$ or non-tumor adjacent tissue $(n=106)$ were obtained from TCGA $(N=1112)$ for validation of the NCI-MD study16S rRNA gene sequencing analysis and results. Due to the fact that all RNA-seq data in TCGA were obtained using poly-A capture, any microbial data from this analysis will necessarily be biased. For this reason, we only used these data as validation of results first identified in our 16S rRNA gene sequencing analysis. Public data, including all clinical patient information (Table 1), was downloaded from the Data Matrix on the TCGA website, https://portal.gdc.cancer.gov. The raw data in the form of BAM and FastQ files were download from a secure server at CGHUB and access was applied for and approved for raw data downloads by University of California Santa Cruz, https://cghub.ucsc.edu/. The files were downloaded and stored in archived format and subsequently un-archived for analysis. The results shown here are in whole or part based upon data generated by the TCGA Research Network: https://gdc.cancer.gov.

\section{DNA extraction and 16S rRNA gene sequencing}

DNA from lung cancer and control lung tissues was isolated according to a tissue-modified version of the standard Human Microbiome Project's DNA isolation procedure. Genomic DNA from frozen lung tissue was extracted after tissue homogenization in Yeast Cell Lysis Buffer (Epicenter) containing lysozyme (Epicenter) by bead beating (TissueLyser II) with proteinase $\mathrm{k}$ (Invitrogen). DNA was purified with the Life Technologies PureLink kit according to the manufacturer's protocol (Invitrogen). A sterile water control (MoBio) was also processed along with all frozen tissue and used as background contamination control for DNA isolation, PCR, and sequencing. Background contamination controls for tissue collection, pathology, and sequencing were also collected through routine swabs after surgery and sequenced in conjunction with tissue samples. Specifically, the NCI-MD study tissues were isolated in a laminar flow hood to minimize contamination for downstream applications, using sterile forceps and gloves. Controls for contamination points during surgical tissue collection and pathological assessment included swabs from inside of the surgical tissue collection vessel before/after, pathology cutting board before/after, pathology knife blade before/after, gloves before/after, and pathology ink bottle rim and collection tube for freezing before/after (Additional file 3). Briefly, swabs were dipped in Yeast cell Lysis buffer and area/object swabbed, then the swab was broken off into tube and frozen at $-80{ }^{\circ} \mathrm{C}$. A negative control was also collected using $50 \mu \mathrm{L}$ of MoBio PCR water as a mock sample (PCR_NC) and processed through DNA extraction with tissues to assess contamination from reagents, which was analyzed on three separate runs of MiSeq. The positive control was the High Even Mock Community (Broad Institute), which was also sequenced on three separate runs of MiSeq. The negative and positive control samples were spiked into four MiSeq runs at a similar concentration to that of the NCI-MD samples. To control for false grouping or batch affects, we randomized the tissue sample types (NT, T, and ImA) (with the exception of $\mathrm{HB}$ controls) across five separate sequencing runs of MiSeq (Additional file 4). The fifth plate consisted of duplicate samples and samples that had failed sequencing on previous runs of MiSeq.

Sequencing for the 16S rRNA gene was performed with $40 \mathrm{ng}$ of sample DNA from 398 cases and 57 controls using primers for variable region V3-V5 with $16 \mathrm{~S}$ rRNA gene sequence-specific portions based on Kozich et al. [54] with adapters for subsequent addition of standard Illumina dual indexes. PCR was performed using a Phusion DNA Polymerase High Fidelity kit (ThermoFisher). The cycling conditions were as follows: $98{ }^{\circ} \mathrm{C}$ for $2 \mathrm{~min}$, then 36 cycles of $98{ }^{\circ} \mathrm{C}$ for $15 \mathrm{~s}, 60{ }^{\circ} \mathrm{C}$ for $1 \mathrm{~min} 40 \mathrm{~s}$, and $74{ }^{\circ} \mathrm{C}$ for $1 \mathrm{~min}$. PCR products were purified using the Agencourt AMPure XP kit according to the manufacturer's instructions (Beckman Coulter). Second round PCR with Illumina dual-index oligos was performed using a Phusion DNA Polymerase High Fidelity kit (ThermoFisher) as following: $98{ }^{\circ} \mathrm{C}$ for $2 \mathrm{~min}$, then six cycles of $98{ }^{\circ} \mathrm{C}$ for $15 \mathrm{~s}, 72{ }^{\circ} \mathrm{C}$ for $20 \mathrm{~s}$, and $72{ }^{\circ} \mathrm{C}$ for $1 \mathrm{~min}$. Samples were pooled and purified using Agencourt 
AMPure XP. Sequencing was conducted on Illumina MiSeq instrument using v3 600 cycles kit (Additional file 1: Supplemental Methods).

\section{Full-length 16S rDNA PCR reactions (PacBio)}

Full-length $16 \mathrm{~S}$ amplifications were performed using: $1 \mu \mathrm{L}$ of total DNA as template; $0.25 \mu \mathrm{M}$ of the universal $16 \mathrm{~S}$ primers F27 and R1492 with four different sets of asymmetric barcodes at (Additional file 1: Table S9). and GoTaq Hot Start Master Mix (Promega) in a $50 \mu \mathrm{L}$ final volume. Cycling conditions were: $94{ }^{\circ} \mathrm{C}, 3 \mathrm{~min}$; 35 cycles of $94{ }^{\circ} \mathrm{C} 30 \mathrm{~s}, 54{ }^{\circ} \mathrm{C} 30 \mathrm{~s}, 72{ }^{\circ} \mathrm{C} 2 \mathrm{~min}$; following by a 5 min final elongation at $72{ }^{\circ} \mathrm{C}$. PCR products were cleaned with AxyPrep ${ }^{\text {ma }}$ MagPCR (Corning Life Sciences) according to the manufacturer's protocol and eluted in $40 \mu \mathrm{L}$ of water. Cleaned PCR products were quantified using the Bio-Rad QX200 droplet digital PCR (Bio-Rad) and QX200 EvaGreen ${ }^{\circ}$ Supermix with primers F357 and R534 (Additional file 1: Table S10) targeting the V3 variable region of $16 \mathrm{~S}$ rDNA. Based on the results, amplicon libraries were normalized to the same concentration before pooling. Pooling was always performed using amplicon libraries with distinct barcodes. Multiplexing was performed with 2-4 libraries per pool.

\section{Pacific biosciences circular consensus sequencing}

Sequencing library construction was accomplished using the Pacific Biosciences (PacBio) SMRTbell ${ }^{\text {TM }}$ Template Prep Kit V1 on the normalized pooled PCR products. Sequencing was performed using the PacBio RS II platform using protocol "Procedure \& Checklist - $2 \mathrm{~kb}$ Template Preparation and Sequencing" (part number 001-143-835- 06). DNA Polymerase Binding Kit P6 V2 was used for sequencing primer annealing and polymerase binding. SMRTbell libraries were loaded onto SMRTcells V3 at a final concentration of $0.0125 \mathrm{nM}$ using the MagBead kit, as determined using the PacBio Binding Calculator software. Internal Control Complex P6 was used for all reactions to monitor sequencing performance. DNA Sequencing Reagent V4 was used for sequencing on the PacBio RS II instrument, which included MagBead loading and stage start. Movie time was $3 \mathrm{~h}$ for all SMRTcells. PacBio sequencing runs were set up using RS Remote PacBio software and monitored using RS Dashboard software. Sequencing performance and basic statistics were collected using $\mathrm{SMRT}^{\circ}$ Analysis Server v2.3.0. De-multiplexing and conversion to FastQ was accomplished using the Reads of Insert (ROI) protocol in the SMRT portal v2.3 software. Only reads with a minimum of five circular passes and a predicted accuracy of 90 (PacBio score) or better were used for further analysis. Each read was labeled in the header with the number of CCS (circular consensus sequence) passes and the sample designation using a custom ruby script, followed by concatenation of all reads into a single file for subsequent filtering and clustering.

\section{Filtering and OTU clustering of $16 \mathrm{~S}$ rRNA gene sequence data}

Initial screening for length and quality using QIIME v 1.9.0 (qiime.org) [55]. Reads containing more than five consecutive low-quality base calls (Phred $<$ Q20), were truncated at the beginning of the low-quality region. Due to the low quality of the majority of R2 reads (Phred $<$ Q20 and $<150$ bp length), we used the R1 reads only for this analysis. Passing sequences were required to have high-quality base calls ( $\geq$ Phred Q20) along a minimum of $75 \%$ of the read length to be included. The average Phred score per read was 34 with $88 \%$ of reads having a Phred score $>30$ (Additional file 1: Supplemental Methods, Figure S1, and Table S2). After primer removal, final sequences containing ambiguous bases (Ns) or lengths $<150$ bp were removed. High quality sequences were then screened for spurious PhiX contaminant using BLASTN with a word size of 16 . Reads were then assessed for chimeras using USEARCH61 (de novo mode, 97\% identity threshold for clustering). Non- chimeric sequences were screened for contaminant chloroplast and mitochondria using the RDP naïve Bayesian classifier, as well as non-specific human genome contaminant using Bowtie2 against the UCSC hg19 reference sequence. Finally, sequences were evaluated for residual contaminants using BLASTN searches of the GreenGenes database (v13.5). Filtered reads included those not matching any reference with at least $70 \%$ identity along $60 \%$ of their length. Exploratory assessment using BLASTN searches against the NCBI NT database indicated the majority unknown contaminant reads were amplified human genome sequence. High-quality passing sequences were subsequently clustered into operational taxonomic units using the open-reference operational taxonomic unit (OTU) picking methodology implemented within QIIME using default parameters and the GreenGenes database (99\% OTUs) supplemented by reference sequences from the SILVA database (v111). Before downstream diversity analyses, the OTU table was rarefied to 5500 sequences per sample. Before diversity analysis, contaminants were removed and again OTUs table rarified to 5500 sequences per sample. Alpha diversity estimators and beta-diversity metrics were computed in QIIME with differential abundance analyses performed in $R$. In order to determine significant differences in beta diversity, we used the adonis function in the $\mathrm{R}$ package vegan to conduct PERMANOVA with Bray Curtis distance and 999 permutations in order to be able to compare across studies. All sequences from the MiSeq and PacBio datasets have been deposited at the following location: http://www.ncbi.nlm.nih.gov/bioproject/320383. See Additional file 1: Supplemental Methods for details 
regarding PacBio sequence processing, and Additional file 5 for complete OTU and Additional file 6 for Pathoscope results.

\section{TCGA RNA-seq data processing and alignment}

In order to analyze all RNA-seq unmapped reads from TCGA lung cancer samples, we developed a custom metagenomic analysis pipeline using (1) MetaPhlAn2, (2) Kraken, and (3) Pathoscope [22]. First, all reads were filtered for quality using Trimmomatic (v0.32, minimum average quality $>20$ over a 5 -bp sliding window, minimum final length ${ }^{3} 28 \mathrm{bp}$ ) and searched for potential PhiX-174 contaminant using Bowtie2. Reads passing this filter were then mapped to the comprehensive NCBI Homo sapiens Annotation (Release 106) using Bowtie2 to remove any human-associated reads. The resulting non-human read set was then taxonomically assigned using (1) MetaPhlAn2, (2) Kraken, and (3) Pathoscope in parallel to evaluate consistency in the resulting profiles. Assignments from each method were aggregated at higher taxonomic levels (genus and species) for downstream statistical comparisons (Additional file 1: Table S2). The results from Pathoscope and its validation in other studies lead us to use these data for the remainder of the downstream analysis.

Alpha diversity estimators and beta-diversity (Bray Curtis) metrics were computed in QIIME using genus and species level assignments with differential abundance analyses performed in R and Stata (v13). Full taxonomic assignments for each sample are provided in Additional file 5.

\section{Statistical analysis and classification of taxa associated with lung cancer}

Statistical analysis and visualization, ANOVA and PCoA, was performed on sequencing quality metrics by population sample type (ImA, HB, NT, and T) (Additional file 1: Figure S1). Alpha- and beta-diversity metrics were computed in QIIME with differential abundance analyses performed in R and Stata (v13). Mann-Whitney tests corrected for multiple testing (Benjamini-Hochberg [FDR]) were used to conduct initial comparisons between tissue type and histological subtype (AD or SCC) followed by multivariable logistic regression controlling for multiple confounders (age, gender, race, smoking status, stage, antibiotic exposure, lung location, average Phred score, and sequencing run) (Additional file 1: Table S11). An additional logistic regression model was constructed to estimate the odds of AD versus SCC for each taxa separately (identified from the initial testing) stratified by TP53 mutation status (wild-type versus mutated) with and interaction term between the taxa and mutation added to the model. See Additional file 1: Supplemental Methods for details of statistical modeling.

\section{TP53 gene sequencing and mutation analysis}

Genomic DNA extracted from lung cancer tissues $(n=$ 107) was submitted for TP53-targeted sequencing using the MiSeq Illumina platform. For mutation analysis, 46 samples were SCC. The assay was targeted at the exons and proximal splice sites. Forward and reverse primers were tailed with Illumina Adapter tags for downstream next-generation sequencing using the BioMark HD System (Fluidigm) and Access Array IFC chips and kits (Fluidigm). PCR products were indexed using an 8-mer oligo barcode. See Additional file 1: Table S3 lists sequences for primers used in the sequencing assay. Sequence results were processed and aligned to human genome and underwent $\mathrm{QC}$ requiring coverage $>100$ reads with the variant (most single nucleotide variants [SNVs] had a read depth in the thousands) and minimum allele frequency $>10 \%$. The 100-level cutoff for coverage allows to detect variations if the tumor fraction $>\sim 20 \%$ with $95 \%$ confidence, under the assumption of a diploid genome. The 10\% allele frequency cutoff is derived from that same consideration. The variants called included all common polymorphisms. Because only the tumor was sequenced, in order to score somatic mutations, those deemed to be germline were filtered out. These included SNVs present in dbSNP with high reported allele frequency (common polymorphisms). Also, SNVs in untranslated regions and introns were not considered, as their somatic status and functional implications are unclear. The presence of putative somatic exonic and splicing variants was corroborated in the TCGA and COSMIC datasets. See Additional file 1: Table S2 for details.

\section{Fluorescent in situ hybridization analysis of Acidovorax}

In order to confirm the presence Acidovorax in lung tumor tissue, fluorescently labeled probes were created for each bacterium. Genus or species-specific bacteria probes were hybridized using tumor tissues in addition to gram stain on each. Tumor tissues from cancer cases were fixed in OCT and sectioned frozen $(10 \mu \mathrm{m})$. Before fixation in $4 \%$ paraformaldehyde, sections were thawed at RT. Sections were washed in PBS and the probe $(2 \mu \mathrm{L})$ was added to $90 \mu \mathrm{L}$ FISH buffer $(0.9 \mathrm{M} \mathrm{NaCl}, 0.02 \mathrm{M}$ Tris $\mathrm{pH} 7.5$, $0.01 \%$ SDS, $20 \%$ formamide). This solution was added to the section $(20-100 \mu \mathrm{L})$ and placed in the hybridization chamber $\left(46{ }^{\circ} \mathrm{C}\right)$ for $3-18 \mathrm{~h}$ depending on probe used. Section were washed twice (wash 1: $0.9 \mathrm{M} \mathrm{NaCl}, 0.02 \mathrm{M}$ Tris pH 7.5, 0.01\% SDS, 20\% formamide; wash 2: $0.9 \mathrm{M}$ $\mathrm{NaCl}, 0.02 \mathrm{M}$ Tris pH 7.5, 0.01\% SDS) and incubated at $48{ }^{\circ} \mathrm{C}$ for $15 \mathrm{~min}$. Slides were then dried for $10 \mathrm{~min}$. Before visualization, DAPI and Vectashield were added to the slides. The probe used for FISH was: Acidovorax (CTT TCG CTC CGT TAT CCC, 5' modification: Alexa Fluor 532). Representative fields were imaged using Zeiss 710 
and a 100X objective for the probe. In addition to two-dimensional (2D) images, $\mathrm{Z}$ stacks were also obtained for each bacterial probe and used to reconstruct three-dimensional (3D) images and movies using Imaris software. Quantification of Acidovorax probe reactivity was conducted using ten 2D fields of two patients. At least 300 cells were counted per patient. Percentage (\%) of cells with perinuclear probe reactivity was quantified using ImagePro Plus 6.0 software (Additional file 1: Figure S8).

\section{Additional files}

Additional file 1: Supplementary methods, Figures S1-S9, and Tables S1-11. (PDF $13408 \mathrm{~kb}$ )

Additional file 2: Video S1. 3D video image of Acidovorax. (MP4 6568 kb)

Additional file 3: Background contamination swabs and relative abundance. (XLSX $145 \mathrm{~kb}$ )

Additional file 4: Distribution of samples across runs of 165 rRNA sequencing. (XLSX $18 \mathrm{~kb}$ )

Additional file 5: Clinical metadata and OTUs for NCI-MD samples. (XLSX $808 \mathrm{~kb}$ )

Additional file 6: Clinical metadata and taxa IDs for TCGA samples. (XLSX $17974 \mathrm{~kb})$

\section{Acknowledgements}

We thank the University of Maryland Cancer Studies Team directed by Dean Mann, for their support, including Steven Schech for the collection of background swabs and specimens.

\section{Funding}

This work was supported by intramural funding from the National Cancer Institute, National Institutes of Health, Bethesda, MD. Work by LG and AV were also supported by the Cancer Prevention Research Program fellowship at the National Cancer Institute, National Institutes of Health, Bethesda, MD.

\section{Availability of data and materials}

All de-identified data from this study will be made available upon reasonable request. Specifically, all sequencing data have been deposited under the bioproject number 320838 and are publicly available at the following location: http://www.ncbi.nlm.nih.gov/bioproject/320383 [56].

\section{Authors' contributions}

$\mathrm{KLG}$, JAS, and CCH conceived of the study, experiments, analyzed data, interpreted results, and participated in writing and review. CD, SC, JO, AIR, WB, MAP, SB, PSM, JEK, SVB, ASB, JPE, JCM, GDE, JAS, and JRW sequenced samples and/or processed sequencing data and mutation analysis. KLG, JRW, $N P, A J V$, and ECP conducted statistical analysis of data. AV, JAB, NM, TC, ANH, TMS, and MRW conducted FISH experiments and interpreted results. EDB and MAK provided assistance with procuring and processing biospecimens and clinical databases. BMR, AHD, and GT provided technical and data interpretation assistance and manuscript review. All authors read and approved the final manuscript.

\section{Ethics approval and consent to participate}

This study was approved by the Institutional Review Board at the NIH and all individuals participating in the NCl-MD case-control study signed informed consents for the collection of biospecimens, personal, and medical information.

\section{Consent for publication}

Not applicable.

\section{Competing interests}

James White is a significant shareholder in the company Resphera Insight Inc. All other authors declare that they have no competing interests.

\section{Publisher's Note}

Springer Nature remains neutral with regard to jurisdictional claims in published maps and institutional affiliations.

\section{Author details}

${ }^{1}$ Laboratory of Human Carcinogenesis, Center for Cancer, Research, National Cancer Institute, National Institutes of Health, 37 Convent Dr., Rm 3068A, MSC 4258, Bethesda, MD 20892-4258, USA. ${ }^{2}$ Resphera Biosciences, Baltimore, MD 21231, USA. ${ }^{3}$ Center for Cancer Research Genomics Core, National Cancer Institute, National Institutes of Health, Bethesda, MD 20892, USA. ${ }^{4}$ Division of Biomedical Statistics and Informatics, Mayo Clinic, Rochester, MN 55905, USA. ${ }^{5}$ Department of Educational Psychology, Baylor University, Waco, TX 97346, USA. 'Laboratory of Experimental Immunology, Center for Cancer Research, National Cancer Institute, National Institutes of Health, Bethesda, MD 20892, USA. ${ }^{7}$ Genetics Branch, Center for Cancer Research, National Cancer Institute, National Institutes of Health Bethesda, Bethesda, MD 20892, USA. ${ }^{8}$ Department of Obstetrics and Gynecology, Mayo Clinic, Rochester, MN, USA. ${ }^{9}$ Microbiome Laboratory, Mayo Clinic, Rochester, MN 55905, USA.

${ }^{10}$ Department of Surgery, Mayo Clinic, Rochester, MN 55905, USA.

${ }^{11}$ Department of Microbiology and Immunology, Center for Genomic Sciences, Institute of Molecular Medicine and Infectious Disease, Drexel University College of Medicine, Philadelphia, PA 19129, USA. ${ }^{12}$ National Human Genome Research Institute, National Institutes of Health, Bethesda, MD 20892, USA. ${ }^{13}$ Jackson Laboratory, Framingham, CT 06032, USA. ${ }^{14}$ Present Address: Nutrition Sciences, Baylor University, Waco, TX 97346, USA.

Received: 19 February 2018 Accepted: 2 August 2018

Published online: 24 August 2018

\section{References}

1. AMC. Cancer Facts and Figures. Atlanta: American Cancer Society; 2014.

2. Sugimura $H$, Yang $P$. Long-term survivorship in lung cancer: a review. Chest. 2006;129:1088-97. https://doi.org/10.1378/chest.129.4.1088

3. Boursi B, Mamtani R, Haynes K, Yang YX. Recurrent antibiotic exposure may promote cancer formation-Another step in understanding the role of the human microbiota? Eur J Cancer. 2015;51:2655-64. https://doi.org/10.1016/j. ejca.2015.08.015

4. Dickson RP, Erb-Downward JR, Martinez FJ, Huffnagle GB. The microbiome and the respiratory tract. Annu Rev Physiol. 2016;78:481-504. https://doi.org/ 10.1146/annurev-physiol-021115-105238

5. Dickson RP, Erb-Downward JR, Huffnagle GB. The role of the bacterial microbiome in lung disease. Expert Rev Respir Med. 2013;7:245-57. https://doi.org/10.1586/ers.13.24

6. Erb-Downward JR, Thompson DL, Han MK, Freeman CM, McCloskey L, Schmidt $L A$, et al. Analysis of the lung microbiome in the "healthy" smoker and in COPD. PLoS One. 2011;6:e16384. https://doi.org/10.1371/journal.pone.0016384

7. Twomey KB, Alston M, An SQ, O'Connell OJ, McCarthy Y, Swarbreck D, et al. Microbiota and metabolite profiling reveal specific alterations in bacterial community structure and environment in the cystic fibrosis airway during exacerbation. PLoS One. 2013;8:e82432. https://doi.org/10.1371/journal.pone. 0082432

8. Heijink $I H$, Brandenburg SM, Postma DS, van Oosterhout AJM. Cigarette smoke impairs airway epithelial barrier function and cell-cell contact recovery. Eur Respir J. 2012;39:419-28. https://doi.org/10.1183/09031936. 00193810

9. Venkataraman A, Rosenbaum MA, Werner JJ, Winans SC, Angenent LT. Metabolite transfer with the fermentation product 2,3-butanediol enhances virulence by Pseudomonas aeruginosa. ISME J. 2014;8:1210-20. https://doi.org/10.1038/ismej.2013.232

10. Shiels MS, Albanes D, Virtamo J, Engels EA. Increased risk of lung cancer in men with tuberculosis in the alpha-tocopherol, beta-carotene cancer prevention study. Cancer Epidemiol Biomark Prev. 2011;20:672-8. https://doi.org/10.1158/1055-9965.EPI-10-1166

11. Kostic AD, Chun E, Robertson L, Glickman JN, Gallini CA, Michaud M, et al. Fusobacterium nucleatum potentiates intestinal tumorigenesis and modulates the tumor-immune microenvironment. Cell Host Microbe. 2013; 14:207-15. https://doi.org/10.1016/j.chom.2013.07.007

12. McCoy AN, Araujo-Perez F, Azcarate-Peril A, Yeh JJ, Sandler RS, Keku TO. Fusobacterium is associated with colorectal adenomas. PLoS One. 2013:8: e53653. https://doi.org/10.1371/journal.pone.0053653 
13. Rubinstein MR, Wang X, Liu W, Hao Y, Cai G, Han YW. Fusobacterium nucleatum promotes colorectal carcinogenesis by modulating E-cadherin/beta-catenin signaling via its FadA adhesin. Cell Host Microbe. 2013;14:195-206. https://doi. org/10.1016/j.chom.2013.07.012

14. Strauss J, Kaplan GG, Beck PL, Rioux K, Panaccione R, Devinney R, et al. Invasive potential of gut mucosa-derived fusobacterium nucleatum positively correlates with IBD status of the host. Inflamm Bowel Dis. 2011;17: 1971-8. https://doi.org/10.1002/ibd.21606

15. Tahara T, Yamamoto E, Suzuki H, Maruyama R, Chung W, Garriga J, et al. Fusobacterium in colonic flora and molecular features of colorectal carcinoma. Cancer Res. 2014;74:1311-8. https://doi.org/10.1158/0008-5472. CAN-13-1865

16. Sears $\mathrm{CL}$, Geis AL, Housseau F. Bacteroides fragilis subverts mucosal biology: from symbiont to colon carcinogenesis. J Clin Invest. 2014;124:4166-72. https://doi.org/10.1172/JCl72334

17. Schwitalla S, Ziegler PK, Horst D, Becker V, Kerle I, Begus-Nahrmann Y, et al. Loss of p53 in enterocytes generates an inflammatory microenvironment enabling invasion and lymph node metastasis of carcinogen-induced colorectal tumors. Cancer Cell. 2013;23:93-106. https://doi.org/10.1016/j.ccr. 2012.11.014

18. Robles Al, Harris CC. Clinical outcomes and correlates of TP53 mutations and cancer. Cold Spring Harb Perspect Biol. 2010;2:a001016. https://doi.org/ 10.1101/cshperspect.a001016

19. Oren M, Rotter V. Mutant p53 gain-of-function in cancer. Cold Spring Harb Perspect Biol. 2010;2:a001107. https://doi.org/10.1101/cshperspect.a001107

20. Trump BF, Valigorsky JM, Dees JH, Mergner WJ, Kim KM, Jones RT, et al. Cellular change in human disease. A new method of pathological analysis. Hum Pathol. 1973:4:89-109.

21. Meadow JF, Altrichter AE, Green JL. Mobile phones carry the personal microbiome of their owners. PeerJ. 2014;2:e447. https://doi.org/10.7717/ peerj.447

22. Byrd AL, Perez-Rogers JF, Manimaran S, Castro-Nallar E, Toma I, McCaffrey T, et al. Clinical PathoScope: rapid alignment and filtration for accurate pathogen identification in clinical samples using unassembled sequencing data. BMC Bioinformatics. 2014;15:262. https://doi.org/10.1186/1471-2105-15-262

23. Huang YJ, Nelson CE, Brodie EL, Desantis TZ, Baek MS, Liu J, et al. Airway microbiota and bronchial hyperresponsiveness in patients with suboptimally controlled asthma. J Allergy Clin Immunol. 2011;127:372-81. e371-373. https://doi.org/10.1016/j.jaci.2010.10.048

24. Zhao J, Schloss PD, Kalikin LM, Carmody LA, Foster BK, Petrosino JF, et al. Decade-long bacterial community dynamics in cystic fibrosis airways. Proc Natl Acad Sci U S A. 2012;109:5809-14. https://doi.org/10.1073/pnas.1120577109

25. Hilty M, Burke C, Pedro H, Cardenas P, Bush A, Bossley C, et al. Disordered microbial communities in asthmatic airways. PLoS One. 2010;5:e8578. https://doi.org/10.1371/journal.pone.0008578

26. Meisel JS, Hannigan GD, Tyldsley AS, SanMiguel AJ, Hodkinson BP, Zheng Q, et al. Skin microbiome surveys are strongly influenced by experimental design. J Invest Dermatol. 2016;136:947-56. https://doi.org/10.1016/j.jid.2016.01.016

27. Pragman AA, Kim HB, Reilly CS, Wendt C, Isaacson RE. The lung microbiome in moderate and severe chronic obstructive pulmonary disease. PLoS One. 2012;7:e47305. https://doi.org/10.1371/journal.pone.0047305

28. Segal LN, Alekseyenko AV, Clemente JC, Kulkarni R, Wu B, Gao Z, et al. Enrichment of lung microbiome with supraglottic taxa is associated with increased pulmonary inflammation. Microbiome. 2013;1:19. https://doi.org/ 10.1186/2049-2618-1-19

29. Morris A, Beck JM, Schloss PD, Campbell TB, Crothers K, Curtis JL, et al. Comparison of the respiratory microbiome in healthy nonsmokers and smokers. Am J Respir Crit Care Med. 2013;187:1067-75. https://doi.org/10. 1164/rccm.201210-19130C

30. Pesch B, Kendzia B, Gustavsson P, Jöckel K-H, Johnen G, Pohlabeln H, et al. Cigarette smoking and lung cancer-relative risk estimates for the major histological types from a pooled analysis of case-control studies. Int J Cancer. 2012;131:1210-9. https://doi.org/10.1002/ijc.27339

31. Cancer Genome Atlas Research Network. Comprehensive genomic characterization of squamous cell lung cancers. Nature. 2012;489:519-25. https://doi.org/http://www.nature.com/nature/journal/v489/n7417/abs/ nature11404.html\#supplementary-information

32. Hutter CM, Mechanic LE, Chatterjee N, Kraft P, Gillanders EM, NCI GeneEnvironment Think Tank. Gene-environment interactions in cancer epidemiology: a National Cancer Institute Think Tank report. Genet Epidemiol. 2013;37:643-57. https://doi.org/10.1002/gepi.21756
33. Wu J, Peters BA, Dominianni C, Zhang Y, Pei Z, Yang L, et al. Cigarette smoking and the oral microbiome in a large study of American adults. ISME J. 2016;10:2435-46. https://doi.org/10.1038/ismej.2016.37

34. Charlson ES, Chen J, Custers-Allen R, Bittinger K, Li H, Sinha R, et al. Disordered microbial communities in the upper respiratory tract of cigarette smokers. PLoS One. 2010;5:e15216. https://doi.org/10.1371/journal.pone. 0015216

35. Yu G, Gail MH, Consonni D, Carugno M, Humphrys M, Pesatori AC, et al. Characterizing human lung tissue microbiota and its relationship to epidemiological and clinical features. Genome Biol. 2016;17:163. https://doi. org/10.1186/s13059-016-1021-1

36. Kim HJ, Kim YS, Kim KH, Choi JP, Kim YK, Yun S, et al. The microbiome of the lung and its extracellular vesicles in nonsmokers, healthy smokers and COPD patients. Exp Mol Med. 2017;49:e316. https://doi.org/10.1038/emm.2017.7

37. Adar SD, Huffnagle GB, Curtis JL. The respiratory microbiome: an underappreciated player in the human response to inhaled pollutants? Ann Epidemiol. 2016;26:355-9. https://doi.org/10.1016/j.annepidem.2016.03.010

38. Chopyk J, Chattopadhyay S, Kulkarni P, Claye E, Babik KR, Reid MC, et al. Mentholation affects the cigarette microbiota by selecting for bacteria resistant to harsh environmental conditions and selecting against potential bacterial pathogens. Microbiome. 2017;5:22. https://doi.org/10.1186/s40168017-0235-0

39. Darmawan $\mathrm{R}$, Nakata H, Ohta H, Niidome T, Takikawa K, Morimura S. Isolation and evaluation of PAH degrading Bacteria. J Bioremed Biodeg. 2015;6:283. https://doi.org/10.4172/2155-6199.1000283

40. Nougayrede JP, Homburg S, Taieb F, Boury M, Brzuszkiewicz E, Gottschalk G, et al. Escherichia coli induces DNA double-strand breaks in eukaryotic cells. Science. 2006;313:848-51. https://doi.org/10.1126/science.1127059

41. Putze J, Hennequin C, Nougayrede JP, Zhang W, Homburg S, Karch H, et al. Genetic structure and distribution of the colibactin genomic island among members of the family Enterobacteriaceae. Infect Immun. 2009;77:4696-703. https://doi.org/10.1128/IAl.00522-09

42. Guerra L, Guidi R, Frisan T. Do bacterial genotoxins contribute to chronic inflammation, genomic instability and tumor progression? FEBS J. 2011;278: 4577-88. https://doi.org/10.1111/j.1742-4658.2011.08125.x

43. Siegl C, Rudel T. Modulation of p53 during bacterial infections. Nat Rev Microbiol. 2015;13:741-8. https://doi.org/10.1038/nrmicro3537

44. Wu S, Rhee KJ, Albesiano E, Rabizadeh S, Wu X, Yen HR, et al. A human colonic commensal promotes colon tumorigenesis via activation of $\mathrm{T}$ helper type $17 \mathrm{~T}$ cell responses. Nat Med. 2009;15:1016-22. https://doi.org/10.1038/nm.2015

45. Ahrendt SA, Hu Y, Buta M, McDermott MP, Benoit N, Yang SC, et al. p53 mutations and survival in stage I non-small-cell lung Cancer: results of a prospective study. J Natl Cancer Inst. 2003;95:961-70. https://doi.org/10. 1093/jnci/95.13.961

46. Sapkota AR, Berger S, Vogel TM. Human pathogens abundant in the bacterial metagenome of cigarettes. Environ Health Perspect. 2010;118:351-6. https:// doi.org/10.1289/ehp.0901201

47. Pauly JL, Paszkiewicz G. Cigarette smoke, bacteria, mold, microbial toxins, and chronic lung inflammation. J Oncol. 2011;2011:819129. https://doi.org/ 10.1155/2011/819129

48. Gleeson K, Eggli DF, Maxwell SL. Quantitative aspiration during sleep in normal subjects. Chest. 1997;111:1266-72.

49. Zhang C, Cleveland K, Schnoll-Sussman F, McClure B, Bigg M, Thakkar P, et al. Identification of low abundance microbiome in clinical samples using whole genome sequencing. Genome Biol. 2015;16:265. https://doi.org/10. 1186/s13059-015-0821-z

50. Zhu L, Jing S, Wang B, Wu K, Shenglin MA, Zhang S. Anti-PD-1/PD-L1 therapy as a promising option for non-small cell lung cancer: a single arm meta-analysis. Pathol Oncol Res. 2016;22:331-9. https://doi.org/10.1007/ s12253-015-0011-z

51. Sivan A, Corrales L, Hubert N, Williams JB, Aquino-Michaels K, Earley ZM, et al. Commensal Bifidobacterium promotes antitumor immunity and facilitates anti-PD-L1 efficacy. Science. 2015;350:1084-9. https://doi.org/10. 1126/science.aac4255

52. Tartour E, Zitvogel L. Lung cancer: potential targets for immunotherapy. Lancet Respir Med. 2013;1:551-63. https://doi.org/10.1016/S22132600(13)70159-0

53. Zheng $Y L$, Loffredo CA, Alberg AJ, Yu Z, Jones RT, Perlmutter $D$, et al. Less efficient g2-m checkpoint is associated with an increased risk of lung cancer in African Americans. Cancer Res. 2005;65:9566-73. https://doi.org/10.1158/ 0008-5472.CAN-05-1003 
54. Kozich JJ, Westcott SL, Baxter NT, Highlander SK, Schloss PD. Development of a dual-index sequencing strategy and curation pipeline for analyzing amplicon sequence data on the MiSeq Illumina sequencing platform. Appl Environ Microbiol. 2013;79:5112-20. https://doi.org/10.1128/aem.01043-13

55. Caporaso JG, Kuczynski J, Stombaugh J, Bittinger K, Bushman FD, Costello EK, et al. QIIME allows analysis of high-throughput community sequencing data. Nat Methods. 2010;7:335-6. https://doi.org/10.1038/nmeth.f.303

56. Sequence read archive. http://www.ncbi.nlm.nih.gov/bioproject/320383.

Ready to submit your research? Choose BMC and benefit from:

- fast, convenient online submission

- thorough peer review by experienced researchers in your field

- rapid publication on acceptance

- support for research data, including large and complex data types

- gold Open Access which fosters wider collaboration and increased citations

- maximum visibility for your research: over $100 \mathrm{M}$ website views per year

At $\mathrm{BMC}$, research is always in progress.

Learn more biomedcentral.com/submissions 